\title{
CONTINUITY FOR MAXIMAL COMMUTATOR OF BOCHNER-RIESZ OPERATORS ON SOME WEIGHTED HARDY SPACES
}

\author{
LIU LANZHE AND TONG QINGSHAN
}

Received 17 May 2004 and in revised form 3 November 2004

We show the boundedness for the commutator of Bochner-Riesz operator on some weighted $H^{1}$ space.

\section{Introduction}

Let $b$ be a locally integrable function. The maximal operator $B_{*, b}^{\delta}$ associated with the commutator generated by the Bochner-Riesz operator is defined by

$$
B_{*, b}^{\delta}(f)(x)=\sup _{r>0}\left|B_{r, b}^{\delta}(f)(x)\right|,
$$

where

$$
B_{r, b}^{\delta}(f)(x)=\int_{R^{n}} B_{r}^{\delta}(x-y) f(y)(b(x)-b(y)) d y
$$

and $\left(B_{r}^{\delta}(\hat{f})\right)(\xi)=\left(1-r^{2}|\xi|^{2}\right)_{+}^{\delta} \hat{f}(\xi)$. We also define that

$$
B_{*}^{\delta}(f)(x)=\sup _{r>0}\left|B_{r}^{\delta}(f)(x)\right|,
$$

which is the Bochner-Riesz operator (see [8]). Let $E$ be the space $E=\{h:\|h\|=$ $\left.\sup _{r>0}|h(r)|<\infty\right\}$, then, for each fixed $x \in R^{n}, B_{r}^{\delta}(f)(x)$ may be viewed as a mapping from $[0,+\infty)$ to $E$, and it is clear that $B_{*}^{\delta}(f)(x)=\left\|B_{r}^{\delta}(f)(x)\right\|$ and $B_{*, b}^{\delta}(f)(x)=$ $\left\|b(x) B_{r}^{\delta}(f)(x)-B_{r}^{\delta}(b f)(x)\right\|$.

As well known, a classical result of Coifman et al. [4] proved that the commutator $[b, T]$ generated by $\operatorname{BMO}\left(R^{n}\right)$ functions and the Calderón-Zygmund operator is bounded on $L^{p}\left(R^{n}\right)(1<p<\infty)$. However, it was observed that $[b, T]$ is not bounded, in general, from $H^{p}\left(R^{n}\right)$ to $L^{p}\left(R^{n}\right)$ and from $L^{1}\left(R^{n}\right)$ to $L^{1, \infty}\left(R^{n}\right)$ for $p \leq 1$. But, if $H^{p}\left(R^{n}\right)$ is replaced by some suitable atomic space $H_{b}^{p}\left(R^{n}\right)$ and $H_{B}^{1}\left(R^{n}\right)$ (see $\left.[1,6,7,9]\right)$, then $[b, T]$ maps continuously $H_{b}^{p}\left(R^{n}\right)$ into $L^{p}\left(R^{n}\right)$ and $H_{B}^{1}\left(R^{n}\right)$ into weak $L^{1}\left(R^{n}\right)$ for $p \in(n /(n+1), 1]$. The main purpose of this paper is to establish the weighted boundedness of the commutators 
related to Bochner-Riesz operator and $\operatorname{BMO}\left(R^{n}\right)$ function on some weighted $H^{1}$ space. We first introduce some definitions (see $[1,6,7,9]$ ).

Definition 1.1. Let $b, w$ be locally integrable functions and $w \in A_{1}$ (i.e., $M w(x) \leq c w(x)$ a.e.). A bounded measurable function $a$ on $R^{n}$ is said to be $(w, b)$-atom if

(i) supp $a \subset B=B\left(x_{0}, r\right)$,

(ii) $\|a\|_{L^{\infty}} \leq w(B)^{-1}$,

(iii) $\int a(y) d y=\int a(y) b(y) d y=0$.

A temperate distribution $f$ is said to belong to $H_{b}^{1}(w)$ if, in the Schwartz distributional sense, it can be written as

$$
f(x)=\sum_{j=1}^{\infty} \lambda_{j} a_{j}(x),
$$

where $a_{j}$ 's are $(w, b)$-atoms, $\lambda_{j} \in \mathbb{C}$, and $\sum_{j=1}^{\infty}\left|\lambda_{j}\right|<\infty$. Moreover, $\|f\|_{H_{b}^{1}(w)} \sim \sum_{j=1}^{\infty}\left|\lambda_{j}\right|$. Definition 1.2. Let $w \in A_{1}$. A function $f$ is said to belong to weighted Block $H^{1}$ space $H_{B}^{1}(w)$ if $f$ can be written as (1.4), where $a_{j}$ 's are $w$-atoms (i.e., $a_{j}$ 's satisfy Definition 1.1(i), (ii), and (iii)' $\left.\int a(y) d y=0\right)$ and $\lambda_{j} \in \mathbb{C}$ with

$$
\sum_{j=1}^{\infty}\left|\lambda_{j}\right|\left(1+\log ^{+} \frac{1}{\left|\lambda_{j}\right|}\right)<\infty .
$$

Moreover, $\|f\|_{H_{B}^{1}(w)} \sim \sum_{j=1}^{\infty}\left|\lambda_{j}\right|\left(1+\log ^{+}\left(\left(\sum_{i}\left|\lambda_{i}\right|\right) /\left|\lambda_{j}\right|\right)\right)$.

Now, we formulate our results as follows.

Theorem 1.3. Let $b \in \operatorname{BMO}\left(R^{n}\right)$ and $w \in A_{1}$. Then the maximal commutator $B_{*, b}^{\delta}$ is bounded from $H_{b}^{1}(w)$ to $L_{w}^{1}\left(R^{n}\right)$ when $\delta>(n-1) / 2$.

Theorem 1.4. Let $b \in \operatorname{BMO}\left(R^{n}\right)$ and $w \in A_{1}$. Then the maximal commutator $B_{*, b}^{\delta}$ is bounded from $H_{B}^{1}(w)$ to $L_{w}^{1, \infty}\left(R^{n}\right)$ when $\delta>(n-1) / 2$.

Theorem 1.5. Let $b \in \operatorname{BMO}\left(R^{n}\right)$ and $w \in A_{1}$. Then the maximal commutator $B_{*, b}^{\delta}$ is bounded from $H^{1}(w)$ to $L_{w}^{1, \infty}\left(R^{n}\right)$ when $\delta>(n-1) / 2$.

\section{Proof of theorems}

Proof of Theorem 1.3. It suffices to show that there exists a constant $C>0$ such that for every $(w, b)$-atom $a$,

$$
\left\|B_{*, b}^{\delta}(a)\right\|_{L_{w}^{1}} \leq C
$$

Let $a$ be a $(w, b)$-atom supported on a ball $B=B\left(x_{0}, R\right)$. We write

$$
\begin{aligned}
\int_{R^{n}}[ & \left.B_{*, b}^{\delta}(a)(x)\right] w(x) d x \\
\quad & =\int_{\left|x-x_{0}\right| \leq 2 R}\left[B_{*, b}^{\delta}(a)(x)\right] w(x) d x+\int_{\left|x-x_{0}\right|>2 R}\left[B_{*, b}^{\delta}(a)(x)\right] w(x) d x \equiv I+I I .
\end{aligned}
$$


For $I$, taking $q>1$, by Hölder's inequality and the $L^{q}$-boundedness of $B_{*, b}^{\delta}$ (see [2]), we see that

$$
I \leq C\left\|B_{*, b}^{\delta}(a)\right\|_{L_{w}^{q}} \cdot w(2 B)^{1-1 / q} \leq C\|a\|_{L_{w}^{q}} w(B)^{1-1 / q} \leq C .
$$

For $I I$, let $b_{0}=\left|B\left(x_{0}, R\right)\right|^{-1} \int_{B\left(x_{0}, R\right)} b(y) d y$, then

$$
\begin{aligned}
I I \leq & \sum_{k=1}^{\infty} \int_{2^{k+1} R \geq\left|x-x_{0}\right|>2^{k} R}\left|b(x)-b_{0}\right| B_{*}^{\delta}(a)(x) w(x) d x \\
& +\sum_{k=1}^{\infty} \int_{2^{k+1} R \geq\left|x-x_{0}\right|>2^{k} R} B_{*}^{\delta}\left(\left(b-b_{0}\right) a\right)(x) w(x) d x=I I_{1}+I I_{2} .
\end{aligned}
$$

For $I I_{1}$, we choose $\delta_{0}$ such that

$$
\frac{n-1}{2}<\delta_{0}<\min \left(\delta, \frac{n+1}{2}\right)
$$

and consider the following two cases.

Case $1(0<r \leq R)$. In this case, note that (see [8])

$$
\left|B^{\delta}(z)\right| \leq C(1+|z|)^{-(\delta+(n+1) / 2)},
$$

we have, for $\left|x-x_{0}\right|>2\left|y-x_{0}\right|$,

$$
\begin{aligned}
\left|B_{r}^{\delta}(a)(x)\right| & \leq C r^{-n} \int_{B\left(x_{0}, R\right)} \frac{|a(y)|}{(1+|x-y| / r)^{\delta+(n+1) / 2}} d y \\
& \leq C|B|^{\left(\delta_{0}+(n+1) / 2\right) / n}\left|2^{k+1} B\right|^{-\left(\delta_{0}+(n+1) / 2\right) / n} w(B)^{-1} .
\end{aligned}
$$

Case $2(r>R)$. In this case, note that

$$
\left|\nabla^{\beta} B^{\delta}(z)\right| \leq C(1+|z|)^{-(\delta+(n+1) / 2)}
$$

for any $\beta=\left(\beta_{1}, \ldots, \beta_{n}\right) \in(\mathbb{N} \cup\{0\})^{n}$ and $\left|x-x_{0}\right|>2\left|y-x_{0}\right|$, where

$$
\nabla^{\beta}=\left(\frac{\partial}{\partial x_{1}}\right)^{\beta_{1}} \cdots\left(\frac{\partial}{\partial x_{n}}\right)^{\beta_{n}}
$$

by the vanishing condition of $a$, we gain

$$
\begin{aligned}
\left|B_{r}^{\delta}(a)(x)\right| & \leq C r^{-(n+1)} \int_{B\left(x_{0}, R\right)} \frac{|a(y)|\left|y-x_{0}\right|}{\left(1+\left|x-x_{0}\right| / r\right)^{\delta+(n+1) / 2}} d y \\
& \leq C|B|^{\left(\delta_{0}+(n+1) / 2\right) / n}\left|2^{k+1} B\right|^{-\left(\delta_{0}+(n+1) / 2\right) / n} w(B)^{-1}
\end{aligned}
$$


Combining Case 1 with Case 2, we obtain

$$
\begin{aligned}
I I_{1} \leq & C \sum_{k=1}^{\infty} \int_{2^{k+1} R \geq\left|x-x_{0}\right|>2^{k} R}\left|b(x)-b_{0}\right||B|^{\left(\delta_{0}+(n+1) / 2\right) / n} \\
& \times\left|2^{k+1} B\right|^{-\left(\delta_{0}+(n+1) / 2\right) / n} w(B)^{-1} w(x) d x \\
\leq & C \sum_{k=1}^{\infty} 2^{-k\left(\delta_{0}+(n+1) / 2\right)} w(B)^{-1} \int_{2^{k+1} R \geq\left|x-x_{0}\right|>2^{k} R}\left|b(x)-b_{0}\right| w(x) d x .
\end{aligned}
$$

Since $w \in A_{1}, w$ satisfies the reverse of Hölder's inequality as follows:

$$
\left(\frac{1}{|B|} \int_{B} w(x)^{p} d x\right)^{1 / p} \leq \frac{C}{|B|} \int_{B} w(x) d x
$$

for any ball $B$ and some $1<p<\infty$ (see[10]). Using the properties of $\mathrm{BMO}\left(R^{n}\right)$ functions (see [10]), and noting $w \in A_{1}$, then

$$
\frac{w\left(B_{2}\right)}{\left|B_{2}\right|} \cdot \frac{\left|B_{1}\right|}{w\left(B_{1}\right)} \leq C
$$

for all balls $B_{1}, B_{2}$ with $B_{1} \subset B_{2}$. Thus, by Hölder's and reverse of Hölder's inequalities for $w \in A_{1}$, we get, for $1 / p+1 / p^{\prime}=1$,

$$
\begin{aligned}
I I_{1} \leq & C \sum_{k=1}^{\infty} 2^{-k\left(\delta_{0}+(n+1) / 2\right)} w(B)^{-1}\left|2^{k+1} B\right|\left(\frac{1}{\left|2^{k+1} B\right|} \int_{2^{k+1} B}\left|b(x)-b_{0}\right|^{p^{\prime}} d x\right)^{1 / p^{\prime}} \\
& \times\left(\frac{1}{\left|2^{k+1} B\right|} \int_{2^{k+1} B} w(x)^{p} d x\right)^{1 / p} \\
\leq & C\|b\|_{\text {BMO }} \sum_{k=1}^{\infty} k 2^{-k\left(\delta_{0}-(n-1) / 2\right)}\left(\frac{w\left(2^{k} B\right)}{\left|2^{k} B\right|} \frac{|B|}{w(B)}\right) \leq C .
\end{aligned}
$$

For $I I_{2}$, similar to the estimate of $I I_{1}$, we obtain

$$
B_{r}^{\delta}\left(\left(b-b_{0}\right) a\right)(x) \leq C\|b\|_{\mathrm{BMO}} w(B)^{-1}|B|^{\left(\delta_{0}+(n+1) / 2\right) / n}\left|x-x_{0}\right|^{-\left(\delta_{0}+(n+1) / 2\right)},
$$

thus

$$
\begin{aligned}
I I_{2} & \leq C\|b\|_{\mathrm{BMO}} \sum_{k=1}^{\infty} w(B)^{-1}|B|^{\left(\delta_{0}+(n+1) / 2\right) / n}\left|2^{k} B\right|^{-\left(\delta_{0}+(n+1) / 2\right) / n} w\left(2^{k} B\right) \\
& \leq C\|b\|_{\mathrm{BMO}} \sum_{k=1}^{\infty} 2^{-k\left(\delta_{0}-(n-1) / 2\right)}\left(\frac{w\left(2^{k} B\right)}{\left|2^{k} B\right|} \frac{|B|}{w(B)}\right) \leq C .
\end{aligned}
$$

This finishes the proof of Theorem 1.3.

To prove Theorem 1.4, we recall the following lemma (see $[5,10])$. 
LEMMA 2.1. Let $w \geq 0$ and $\left\{g_{k}\right\}$ be a sequence of measurable functions satisfying

$$
\left\|g_{k}\right\|_{L_{w}^{1, \infty}} \leq 1
$$

Then, for every numerical sequence $\left\{\lambda_{k}\right\}$,

$$
\left\|\sum_{k} \lambda_{k} g_{k}\right\|_{L_{w}^{1, \infty}} \leq C \sum_{k}\left|\lambda_{k}\right|\left(+\log \left(\sum_{j}\left|\lambda_{j}\right| /\left|\lambda_{k}\right|\right)\right) .
$$

Proof of Theorem 1.4. By Lemma 2.1, it is enough to show that there exists a constant $C$ such that

$$
\left\|B_{*, b}^{\delta}(a)\right\|_{L_{w}^{1, \infty} \leq C} \text { for each } w \text {-atom } a \text {. }
$$

Let $a$ be a $w$-atom supported on a ball $B=B\left(x_{0}, r\right)$. We write

$$
\begin{aligned}
w(\{x & \left.\left.\in R^{n}: B_{*, b}^{\delta}(a)(x)>\lambda\right\}\right) \\
& \leq w\left(\left\{x \in 2 B: B_{*, b}^{\delta}(a)(x)>\lambda\right\}\right)+w\left(\left\{x \in(2 B)^{c}: B_{*, b}^{\delta}(a)(x)>\lambda\right\}\right)=I+I I .
\end{aligned}
$$

For $I$, by the $L^{q}$-boundedness of $B_{*, b}^{\delta}$ for $q>1$, we gain

$$
\begin{aligned}
I & \leq \lambda^{-1}\left\|B_{*, b}^{\delta}(a) \chi_{2 B}\right\|_{L_{w}^{1}} \leq C \lambda^{-1}\left\|B_{*, b}^{\delta}(a)\right\|_{L_{w}^{q}} \cdot w(B)^{1-1 / q} \\
& \leq C \lambda^{-1}\|a\|_{L_{w}^{q}} \cdot w(B)^{1-1 / q} \leq C \lambda^{-1} .
\end{aligned}
$$

For $I I$, let $b_{0}=|B|^{-1} \int_{B} b(x) d x$, notice that

$$
\begin{aligned}
B_{*, b}^{\delta}(a)(x) & =\left\|b(x) B_{r}^{\delta}(a)(x)-B_{r}^{\delta}(b a)(x)\right\| \\
& =\left\|\left(b(x)-b_{0}\right) B_{r}^{\delta}(a)(x)-B_{r}^{\delta}\left(\left(b-b_{0}\right) a\right)(x)\right\| \\
& \leq\left\|\left(b(x)-b_{0}\right) B_{r}^{\delta}(a)(x)\right\|+\left\|B_{r}^{\delta}\left(\left(b-b_{0}\right) a\right)(x)\right\| \\
& \leq\left|b(x)-b_{0}\right| B_{*}^{\delta}(a)(x)+B_{*}^{\delta}\left(\left(b-b_{0}\right) a\right)(x),
\end{aligned}
$$

we have

$$
\begin{aligned}
I I \leq & w\left(\left\{x \in(2 B)^{c}:\left|b(x)-b_{0}\right| g_{\mu}^{*}(a)(x)>\frac{\lambda}{2}\right\}\right) \\
& +w\left(\left\{x \in(2 B)^{c}: g_{\mu}^{*}\left(\left(b-b_{0}\right) a\right)(x)>\frac{\lambda}{2}\right\}\right)=I I_{1}+I I_{2} .
\end{aligned}
$$

Similar to the proof of Theorem 1.3, we get

$$
\begin{aligned}
I I_{1} & \leq C \lambda^{-1} \int_{(2 B)^{c}}\left|b(x)-b_{0}\right| B_{*}^{\delta}(a)(x) w(x) d x \\
& =C \lambda^{-1} \sum_{k=1}^{\infty} \int_{2^{k+1} B \backslash 2^{k} B}\left|b(x)-b_{0}\right| B_{*}^{\delta}(a)(x) w(x) d x \leq C \lambda^{-1}\|b\|_{\mathrm{BMO}}, \\
I I_{2} & \leq C \lambda^{-1} \int_{(2 B)^{c}} B_{*}^{\delta}\left(\left(b-b_{0}\right) a\right)(x) w(x) d x \leq C \lambda^{-1}\|b\|_{\mathrm{BMO}} .
\end{aligned}
$$


Combining the estimate of $I, I I_{1}$, and $I I_{2}$, we gain

$$
w\left(\left\{x \in R^{n}: B_{*, b}^{\delta}(a)(x)>\lambda\right\}\right) \leq C \lambda^{-1}\|b\|_{\mathrm{BMO}} .
$$

This completes the proof of Theorem 1.4.

Proof of Theorem 1.5. . Given $f \in H^{1}(w)$, let $f=\sum_{j} \lambda_{j} a_{j}$ be the atomic decomposition for $f$. By a limiting argument, it suffices to show Theorem 1.5 for a finite sum of $f=$ $\sum_{Q} \lambda_{Q} a_{Q}$ with $\sum_{Q}\left|\lambda_{Q}\right| \leq C\|f\|_{H^{1}(w)}$. We may assume that each $Q$ (the supporting cube of $a_{Q}$ ) is dyadic. For $\lambda>0$ by [3, Lemma 4.1], there exists a collection of pairwise disjoint dyadic cubes $\{S\}$ such that

$$
\begin{gathered}
\sum_{Q \subset S}\left|\lambda_{Q}\right| \leq C \lambda|S|, \quad \forall S, \\
\sum_{S}|S| \leq \lambda^{-1} \sum_{Q}\left|\lambda_{Q}\right|, \quad\left\|\sum_{Q \not \subset S} \lambda_{Q}|Q|^{-1} \chi_{Q}\right\|_{L^{\infty}} \leq C \lambda .
\end{gathered}
$$

Let $E=\bigcup_{S} \bar{S}$, where for a fixed cube $Q, \bar{Q}$ denotes the cube with the same center as $Q$ but with the side-length $4 \sqrt{n}$ times that of $Q$. Then, $|E| \leq C \lambda^{-1}\|f\|_{H^{1}}$. Set $M(x)=$ $\sum_{S} \sum_{Q \subset S} \lambda_{Q} a_{Q}, N(x)=f(x)-M(x)$. By the $L^{2}$ boundedness of $B_{*, b}^{\delta}$ and the well-known argument, it suffices to show that

$$
w\left(\left\{x \in E^{c}: B_{*, b}^{\delta}(M)(x)>\lambda\right\}\right) \leq C \lambda^{-1}\|f\|_{H^{1}(w)} .
$$

Because $B_{*, b}^{\delta}(M)(x) \leq \sum_{S} \sum_{Q \subset S}\left|\lambda_{Q}\right| B_{*, b}^{\delta}\left(a_{Q}\right)(x)$, we have

$$
\begin{aligned}
w(\{x & \left.\left.\in E^{c}: B_{*, b}^{\delta}(M)(x)>\lambda\right\}\right) \\
& \leq C \lambda^{-1} \int_{E^{c}} B_{*, b}^{\delta}(M)(x) w(x) d x \\
& \leq C \lambda^{-1} \sum_{S} \sum_{Q \subset S}\left|\lambda_{Q}\right| \sum_{k=1}^{\infty} \int_{2^{k+1} \bar{Q} \backslash 2^{k} \bar{Q}} B_{*, b}^{\delta}\left(a_{Q}\right)(x) w(x) d x
\end{aligned}
$$

similar to the estimate of Theorem 1.3, we get, when $x \in E^{c}$,

$$
\begin{aligned}
B_{*, b}^{\delta}\left(a_{\mathrm{Q}}\right)(x) \leq & C\|b\|_{\mathrm{BMO}} w(B)^{-1}|Q|^{\left(\delta_{0}+(n+1) / 2\right) / n}\left|x-x_{0}\right|^{-\left(\delta_{0}+(n+1) / 2\right)} \\
& +C\left|b(x)-b_{0}\right| w(B)^{-1} 2^{-k\left(\delta_{0}+(n+1) / 2\right)}
\end{aligned}
$$


thus, by Hölder's and reverse of Hölder's inequalities for $w \in A_{1}$, we obtain

$$
\begin{aligned}
w(\{x & \left.\left.\in E^{c}: B_{*, b}^{\delta}(M)(x)>\lambda\right\}\right) \\
& \leq C \lambda^{-1} w(B)^{-1} \sum_{S} \sum_{Q \subset S}\left|\lambda_{Q}\right| \sum_{k=1}^{\infty} k 2^{-k\left(\delta_{0}+(n+1) / 2\right)} w\left(2^{k} Q\right) \\
& \leq C \lambda^{-1} \sum_{S} \sum_{Q \subset S}\left|\lambda_{Q}\right| \sum_{k=1}^{\infty} k 2^{-k\left(\delta_{0}-(n-1) / 2\right)} \\
& \leq C \lambda^{-1} \sum_{S} \sum_{Q \subset S}\left|\lambda_{Q}\right| \leq C \lambda^{-1}\|f\|_{H^{1}(w)} .
\end{aligned}
$$

This finishes the proof of Theorem 1.5.

\section{Acknowledgment}

The author would like to express his gratitude to the referee for his very valuable comments and suggestions.

\section{References}

[1] J. Álvarez, Continuity properties for linear commutators of Calderón-Zygmund operators, Collect. Math. 49 (1998), no. 1, 17-31.

[2] J. Álvarez, R. J. Bagby, D. S. Kurtz, and C. Pérez, Weighted estimates for commutators of linear operators, Studia Math. 104 (1993), no. 2, 195-209.

[3] M. Christ, Weak type $(1,1)$ bounds for rough operators, Ann. of Math. (2) 128 (1988), no. 1, 19-42.

[4] R. R. Coifman, R. Rochberg, and G. Weiss, Factorization theorems for Hardy spaces in several variables, Ann. of Math. (2) 103 (1976), no. 3, 611-635.

[5] G. Hu and S. Lu, The commutator of the Bochner-Riesz operator, Tohoku Math. J. (2) 48 (1996), no. 2, 259-266.

[6] Y. Komori, Weak type estimates for commutators of singular integral operators, to appear in Sci. Math. Japoncae.

[7] Weighted $H^{1}\left(\mathbf{R}^{n}\right)$ estimates for commutators of singular integral operators, Far East J. Math. Sci. (FJMS) 3 (2001), no. 6, 889-898.

[8] S. Z. Lu, Four Lectures on Real Hp Spaces, World Scientific Publishing, New Jersey, 1995.

[9] C. Pérez, Endpoint estimates for commutators of singular integral operators, J. Funct. Anal. 128 (1995), no. 1, 163-185.

[10] E. M. Stein, Harmonic Analysis: Real-Variable Methods, Orthogonality, and Oscillatory Integrals, Princeton Mathematical Series, vol. 43, Princeton University Press, New Jersey, 1993.

Liu Lanzhe: College of Mathematics and Computer, Changsha University of Science and Technology, Changsha 410077, China

E-mail address: lanzheliu@263.net

Tong Qingshan: College of Mathematics and Computer, Changsha University of Science and Technology, Changsha 410077, China 


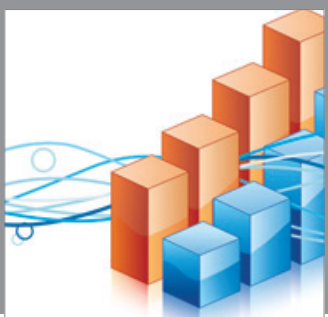

Advances in

Operations Research

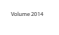

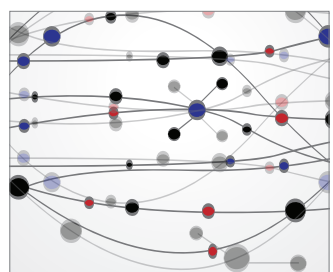

\section{The Scientific} World Journal
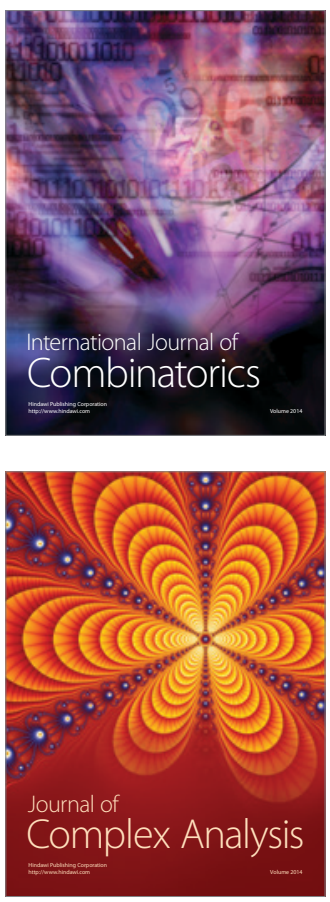

International Journal of

Mathematics and

Mathematical

Sciences
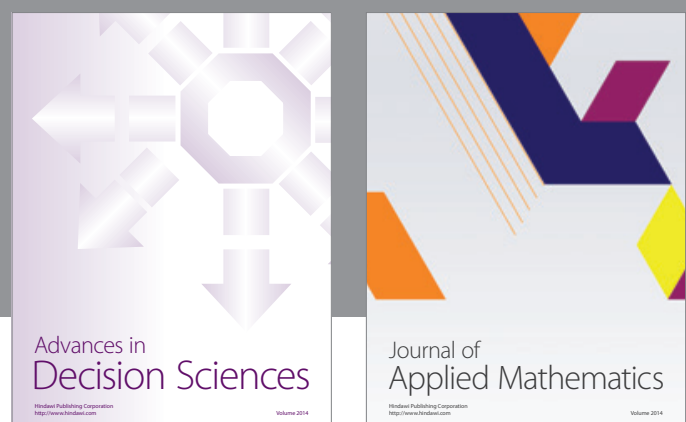

Journal of

Applied Mathematics
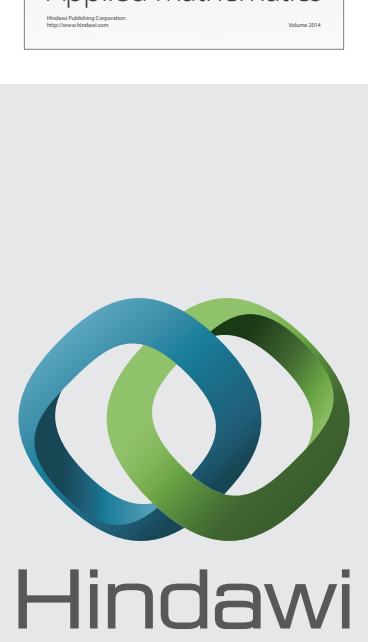

Submit your manuscripts at http://www.hindawi.com
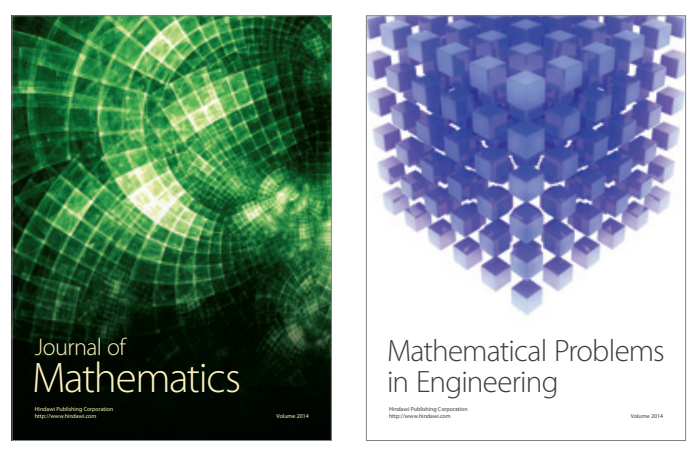

Mathematical Problems in Engineering
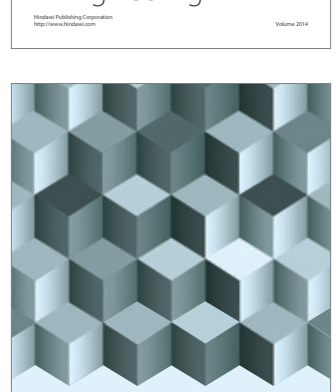

Journal of

Function Spaces
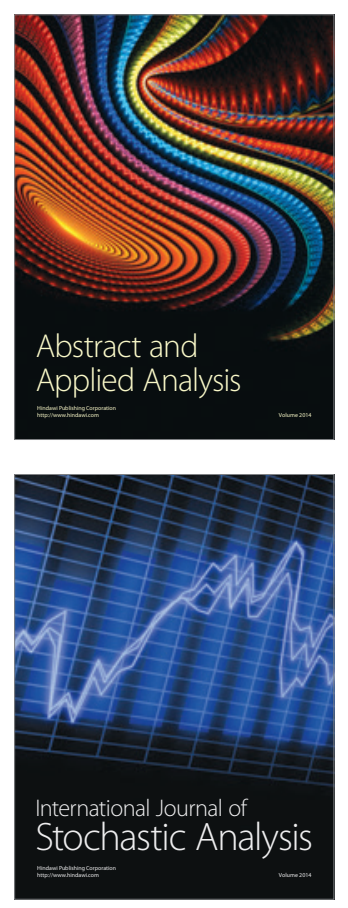

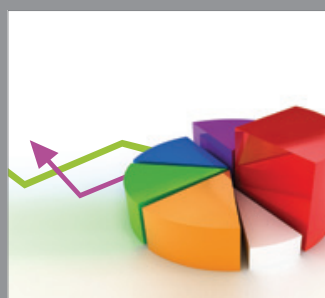

ournal of

Probability and Statistics

Promensencen
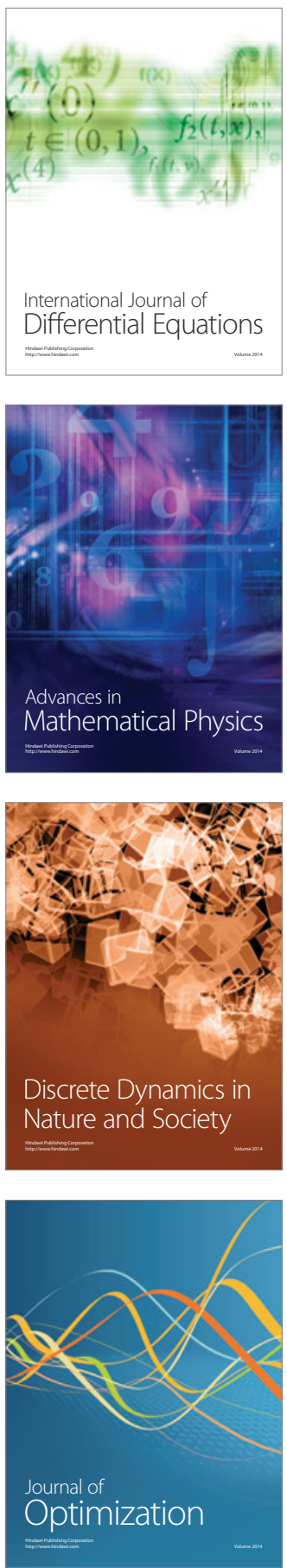